\title{
La importancia de comunicar el servicio de mediación
}

KARINA E. BATTOLA, UNIVERSIDAD NACIONAL DE CÓRDOBA, ARGENTINA

Fecha de recepción: abril de 2014

Fecha de aceptación: agosto de 2014

\section{RESUMEN}

El objetivo de este planteo es destacar la importancia de comunicar el servicio de mediación como método no adversarial de resolución de conflictos. Informar estas cuestiones a la sociedad supone dar a conocer la existencia de medios no violentos de solución de las controversias, poniendo así a disposición de la comunidad información relevante que contribuye a la pacificación social.

Distintos espacios pueden ocuparse de la comunicación de este mecanismo pacífico de abordaje de los conflictos. Entre ellos, se focaliza en primer lugar, los organismos del Estado encargados de la promoción de políticas públicas concernientes al fomento y desarrollo de distintas herramientas que posibilitan solucionar los conflictos jurídicosociales; en segundo lugar, el espacio curricular que ofrece el ámbito universitario mediante actividades de extensión, de divulgación científica o de práctica jurídica; $y$, por último, la comunidad de mediadores, cuyo rol es vital para la promoción de cambios propicios que facilitan la construcción de respuestas pacíficas a las relaciones sociales conflictivas.

PALABRAS CLAVE: servicio de mediación, comunicabilidad de la mediación, método no adversarial de resolución de disputas, conflicto social y comunicación, política pública.

\section{ABSTRACT}

This piece highlights the importance of communicating the mediation service as a nonadversarial method of dispute resolution. To report these issues to society supposes to disclose the existence of non-violent means of resolving disputes and making them available to the community. This information is relevant and contributes to social peace. There are different agents that may communicate such peaceful approach to conflict. 
Among them, I first mention state agencies responsible for the promotion of public policies concerning various tools that allow the solving of legal and social conflicts; second, the curricular space offered by the university and its links with society at large, through scientific or legal practice dissemination; and finally, community mediators, who hold a key role in the promotion of favorable changes that facilitate building peaceful approaches between conflicting social forces.

KEYWORDS: mediation service, communication of mediation, non-adversarial method of dispute resolution, communication and social conflict, public policy.

\section{INTRODUCCIÓN}

La actividad de difusión posee características propias de las actividades de extensión. El objetivo de este artículo es destacar la importancia de comunicar el servicio de mediación como método no adversarial de resolución de conflictos.

La fundamentación de esto radica en el interés de dar a conocer a la sociedad la existencia de este mecanismo de abordaje de problemáticas que se presentan en las relaciones sociales -sean éstas de naturaleza civil, familiar, comunitaria, etc. $-y$, al mismo tiempo, informar que la mediación se caracteriza por ser un medio no violento de solución de las controversias. Con ello, se pone a disposición de la comunidad información relevante para la pacificación social.

Cabe destacar que distintos espacios pueden ocuparse de la comunicación dela mediación como método pacífico de abordaje de los conflictos. Entre ellos se focalizan los siguientes:

En primer lugar, los organismos del Estado encargados de la promoción de políticas públicas concernientes al fomento y desarrollo de distintas herramientas para la solución de los conflictos jurídicos-sociales.

En segundo lugar, el espacio curricular que ofrece el ámbito universitario mediante actividades de extensión, de divulgación científica o de práctica jurídica.

Por último, la comunidad de mediadores, cuyo rol es vital para la promoción de cambios propicios que facilitan la construcción de respuestas pacíficas a las relaciones sociales conflictivas.

La importancia dela comunicación del servicio de mediación se advierteen los beneficios que reviste el conocimiento de este método en el espacio donde se producen y se multiplican los conflictos, contribuyendo a una mejor comunicación interpersonal o intergrupal sobre las relaciones conflictivas.

Resulta oportuno destacar que los métodos alternativos de resolución de conflictos ocupan un lugar relevante en el sistema de administración de justicia. 
Estos métodos implican la gestión de las situaciones de controversia de manera dialogada, sin coerción y de manera integral, reconociendo la capacidad de las personas de poder abordar la problemática en la que están involucradas, así como también encontrar soluciones que satisfagan sus propios intereses.

\section{ORGANISMOS DEL ESTADO ENCARGADOS DE LA PROMOCIÓN DE POLÍTICAS PÚBLICAS CONCERNIENTES AL FOMENTO Y DESARROLLO DE MÉTODOS PACÍFICOS DE SOLUCIÓN DE CONFLICTOS}

Forma parte de las obligaciones del Estado moderno y democrático ocuparse del bienestar social, promoviendo el acceso a la justicia. Para esto, no basta con la tutela de los derechos de los ciudadanos y con un Poder Judicial organizado, sino que se requiere que se pongan a disposición de la sociedad otros mecanismos de solución de conflictos que resulten más eficientes y menos costosos en términos económicos y en relación al tiempo requerido para la solución de las controversias.

De este modo, adquiere relevancia que el Estado difunda los distintos métodos de abordaje de las problemáticas de los individuos, a los fines de que los ciudadanos puedan acceder al conocimiento de estas temáticas. Poner al alcance de la comunidad el derecho que existe y que se encuentra vigente permite el acceso a la mediación en virtud del conocimiento que se obtuvo al respecto. Sin lugar a dudas, la comunicación de este mecanismo previsto en el ordenamiento jurídico es prioritaria para que el ciudadano pueda tomar una decisión informada sobre la utilización de los métodos que implica.

En esta línea de análisis, es oportuno poner de manifiesto que el Gobierno de la provincia de Córdoba, Argentina, organizó en el año 2013, la Primera Feria Provincial de "productos y servicios públicos", denominada "MuniFeria Provincial 2013". El mencionado evento se llevó a cabo en un espacio público con la distribución de distintos stands institucionales -cada uno de ellos con una extensión de 100 metros cuadrados-destinados a la comunicación de los productos y servicios propios de las agencias pertenecientes a la Provincia de Córdoba.

Así, el Ministerio de Justicia y Derechos Humanos de dicha provincia dispuso de un espacio para la difusión de los servicios que prestan las diferentes áreas que lo componen. Una de esas áreas es la Dirección de Métodos Alternativos de Resolución de Conflictos, la cual proyectó la divulgación del mecanismo de la mediación logrando, con ello, la difusión anhelada y la interacción con los visitantes que recibieron información adecuada sobre la mediación. Además, resulta relevante destacar la participación de algunos mediadores del ámbito local que prestaron su colaboración para esta actividad, tanto para el diseño de la misma como también para formar parte del cuerpo de agentes que ofrecerían comunicación a las personas asistentes a la feria. 
La tarea de difusión se realizó mediante diferentes recursos, entre los que se pueden mencionar: presentaciones orales con apoyos técnicos visuales, entrega de folletos y filmaciones breves de situaciones de mediación puntuales, en las que se resaltaron escenas de discurso inicial y relatos de las partes, que han sido filmados con actores en virtud de la confidencialidad que caracteriza el procedimiento de mediación.

Lederach(2000,p. 22) sostiene que la paz no se ve solamente como una fase en el tiempo o una condición; es un proceso social dinámico y como tal requiere "construcción", que conlleva inversión, materiales, diseño arquitectónico, coordinación del trabajo, colocación de los materiales y trabajo de acabado, además de un mantenimiento continuo.

En este sentido, las actividades de comunicación del servicio de mediación se enmarcan en el fortalecimiento de la paz social, al facilitar la construcción de respuestas pacíficas a las relaciones sociales conflictivas.

\section{ESPACIO CURRICULAR EN EL ÁMBITO UNIVERSITARIO MEDIANTE ACTIVIDADES DE EXTENSIÓN, DE DIVULGACIÓN CIENTÍFICA O DE PRÁCTICA JURÍDICA}

Resulta relevante la conexión entre la comunidad educativa y la sociedad. Así, la comunicación de la mediación como método alternativo de solución de conflictos por parte de los espacios universitarios posibilita el conocimiento de otros modos pacíficos de gestión de las problemáticas.

La comunicación del servicio de mediación importa un fenómeno complejo que vincula a las instituciones-entre ellas la universidad-con los individuos que conforman la sociedad. El objetivo de proponer comunicar los métodos alternativos de resolución de conflictos, desde el ámbito universitario, es ofrecer, en primer lugar, una formación dirigida a los alumnos que contribuya a la utilización de la mediación como modo pacíficos de gestión de controversias $\mathrm{y}$, en segundo lugar, demostrar la aplicación de este instrumento que permite el diálogo y la tolerancia para la convivencia en sociedad.

Lo mencionado precedentemente puede implementarse mediante actividades de extensión, de divulgación o de la práctica profesional inserta en los planes de estudios de los alumnos universitarios.

En el ámbito local -precisamente en la Facultad de Derecho y Ciencias Sociales de la Universidad Nacional de Córdoba-en el año2000, se incorpora la asignatura "Teoría del Conflicto y la Decisión. Métodos de Resolución de Conflictos" dentro de la currícula de grado de los alumnos de abogacía ${ }^{1}$.

Asimismo, a partir del año 2010, se lleva adelante una experiencia de divulgación de este instrumento en el espacio curricular de la Práctica Profesional. Dichaactividadimplica que los alumnosdela carrera deAbogacía puedanadvertir

1 Esta es una nota distintiva, atento a que los planes de estudios de dicha carrera en otras jurisdicciones provinciales incorporan algunos temas a los que se refiere la asignatura de las materias de Derecho Procesal. 
problemáticas que se presentan en diferentes entidades. A partir de ello, se lleva adelante el proceso de enseñanza-aprendizaje, en el que el docente responsable asignado a esta labor brinda a los estudiantes la capacitación y tutoría referidas a la temática de mediación. Luego, se produce un contacto con las instituciones receptoras de esta actividad para ofrecerles información necesaria sobre la existencia de modos no litigiosos previstos por el Derecho ${ }^{2}$.

Así, este espacio curricular obligatorio de la carrera de Abogacía tiene características tanto de transferencia de conocimiento como de extensión, en tanto permite divulgar el conocimiento sobre la mediación a las distintas instituciones ubicadas en la Provincia de Córdoba de la República Argentina.

A los fines de mencionar el proyecto denominado "Alfabetización sobre métodos alternativos de resolución de conflictos. Mediación", cabe destacar que se promueve el desarrollo de habilidades actitudinales de divulgación por parte de los alumnos de la carrera de Abogacía, se identifican instituciones o entidades en las que se pueden presentar situaciones de conflicto (Entelman, 2002) y se proyectan encuentros con los actores de estas instituciones, informando sobre el servicio de mediación.

Cabe señalar que el planteo mencionado genera beneficios tanto para los estudiantes que son formados en la utilización de medios pacíficos de gestión de controversias contemplados en el ordenamiento jurídico, como parala comunidad que obtiene los conocimientos necesarios sobre la existencia del servicio de mediación y los lugares habilitados para solicitar esta herramienta de solución de conflictos.

La tarea de formación en métodos no adversariales de resolución de controversias, dirigida a futuros operadores jurídicos, supone un aporte relevante a la práctica del Derecho. Al mismo tiempo, la actividad de transferencia de conocimientos del servicio de mediación implica poner a disposición de las personas nociones adecuadas que posibiliten participación, tolerancia y diálogo, en situaciones en que sea preciso gestionar la conflictividad.

\section{EL ROL DE LOS MEDIADORES EN LA PROMOCIÓN DE CAMBIOS PROPICIOS QUE FACILITAN LA CONSTRUCCIÓN DE RESPUESTAS PACÍFICAS A LAS RELACIONES SOCIALES CONFLICTIVAS}

Se destaca la relevancia de la labor de la comunidad demediadores en la contribución que realizan en la sociedad, al generar un compromiso social mediante esfuerzos que aportan al diálogo, a la tolerancia y a la cultura de la pacificación social.

Es por ello que el mediador debe ejercer su rol profesional consciente del alcance restaurativo de la mediación en el ámbito penal, para lo cual actuará nutrido de sus conocimientos tanto sobre conflictología y procesos de decisión,

2 Actualmente la autora se desempeña como docente-tutor del proyecto "Alfabetización en métodos alternativos de resolución de conflictos" correspondiente al espacio curricular Práctica Profesional III, de la Facultad de Derecho y Ciencias Sociales de la Universidad Nacional de Córdoba. 
como sobre los presupuestos de Justicia Restaurativa. Estas cuestiones conforman el "saber" del mediador y otorgan sustento a las competencias necesarias que aplica, es decir, el "saber hacer" por el cual se ponen de manifiesto las habilidades relativas a su rol.

La permanente formación teórica y la continua experiencia de la práctica profesional fortalecen el desempeño del mediador en la gestión de las situaciones de conflictos en el marco de un modelo de Justicia Restaurativa.

Resulta oportuno señalar que entre las diversas actividades de difusión que pueden realizar los mediadores, se encuentra la tarea de comunicar el servicio de mediación, mediante espacios de divulgación como son por ejemplo columnas de diarios locales dispuestos a colaborar con este desafío. En este sentido, cabe destacar que existe una trayectoria que data de once años - desde el año 2002-, sostenida de manera conjunta entre editores del diario Comercio y Justicia y mediadores de la Provincia de Córdoba, Argentina, quienes se encargan de escribir-de manera periódica-contenidos concretos y casos prácticos sobre mediación a los fines de divulgar los beneficios y jerarquizar esa herramienta de resolución de conflictos ${ }^{3}$.

Como producto del compromiso asumido tanto por los mediadores como por los editores del diario mencionado, surge en el año 2013 el libro Casos y Cosas de Mediación. Dicho libro reúne temas y experiencias de casos reales de mediación, mediante una compilación de artículos oportunamente publicados en Comercio y Justicia, realizada por Samuel Paszuki. Así, el libro contiene en la primer parte-capítulos 1 a 3-temas sobre lenguaje, comunicación, conflicto, emociones, percepciones; en la segunda parte-capítulos 4 a 6-se agrupan reflexiones sobre el poder, el reconocimiento, la interdisciplina por la misma complejidad-en términos de Morin (1990) que implican los procesos demediación; en la tercera parte-capítulos 7 a 13-se reúnen artículos que refieren a conflictos de distinta naturaleza, esto es, daños y perjuicios, penales, comunitario, empresarial, todo ello en base a aportes doctrinarios como los de Bianchi (1996) yCalcaterra (2002); en la cuarta parte-capítulos 14 a 20 se hace mención a casos de mediación vinculados con temática de relaciones de familia. Finalmente, en la última parte se describe una valiosa experiencia en mediación comunitaria. Esta compilación es, sin lugar a dudas y tal como lo señala Marinés Suares en el prólogo del libro, comunicar la mediación como un proceso en crecimiento. (Paszucki, 2013)

Resulta relevante destacar la necesidad de continuar ejecutando acciones adecuadas para un cambio social que aspire a la pacificación social. Es factible que los mediadores puedan asumir el compromiso de un protagonismo activo, tanto en la difusión del significado y finalidades de la mediación como en las potencialidades que emergen de este procedimiento, con el fin de estimular la

3 Dicha actividad es coordinada por el mediador Samuel Paszucki, quien recibe los aportes del resto de los mediadores y es nexo con el editor de la columna del diario mencionado. 
gestión de los conflictos mediante herramientas que contribuyan a la consolidación de la paz social.

En este sentido, la comunidad de mediadores tiene también a su alcance distintas posibilidades, entre las que se puede mencionar las siguientes:

Proponer que los establecimientos educativos de los distintos niveles incorporen en sus planes de estudio asignaturas relacionadas con el conflicto y los métodos alternativos de resolución de los mismos.

Aportes teóricos sobre la temática de mediación que realizan los propios mediadores, tales como Battola (2013), Eiras Nordenstahl (2010) y Álvarez (1995), entre muchos otros.

Presentar proyectos referidos al estudio de la Justicia Restaurativa en las unidades académicas de Derecho y Ciencias Sociales y posteriormente divulgar los resultados de dichos proyectos.

Participar en reformas legislativas que contemplen el procedimiento de mediación en conflictos de índole penal.

Cada una de estas temáticas mencionadas, así como también cualquier otra que se pudiera esbozar en esta línea, implica cimentar las bases que facilitan la producción de cambios para un crecimiento sustentable en aporte a la contribución de la pacificación social. Es por ello, que resulta necesario asumir el desafío de generar pensamientos que enriquezcan la temática y que aporten utilidades para la práctica pacífica de la gestión de conflictos, como así también, reflexionar sobre el compromiso de los mediadores de continuar trabajando para la paz.

\section{RELATOS DE CASOS PARA COMUNICAR EL SERVICIO DE MEDIACIÓN}

Entre las distintas experiencias en el procedimiento de mediación, se seleccionan con fines ilustrativos dos casos en cuyos relatos se procura compartir el mecanismo de trabajo que se lleva adelante en este modo no adversarial de resolución de conflictos.

El primero de los casos hace referencia a una situación conflictiva de índole familiar y, el segundo, a una derivación a mediación de un conflicto de naturaleza penal.

Primer relato: Un caso de mediación de índole familiar ${ }^{4}$. Llega a la primera reunión una señora (Marta) con dificultades para caminar, incluso se ayudaba con un bastón, acompañada por un joven adolescente, su hijo más chico de 16 años (Ignacio). Todo lo que sabíamos era que se había derivado de Asesoría de Familia por cuestiones referidas a las relaciones de familia.

Marta nos cuenta que había convocado a sus tres hijos mayores, pues pade-

4 El relato del caso es realizado por las mediadoras Karina E. Battola y Laura San Millán, intervinientes en una mediación realizada durante el año 2013. 
cía una enfermedad neurológica degenerativa progresiva y necesitaba que sus hijos le ayudaran tanto económicamente, como en su cuidado personal, pues había actividades que ya no podía realizar sola. Agrega que Ignacio la ayudaba en lo que podía, pero que era chico y además cursaba sus estudios secundarios y que ella cobraba una pensión de $\$ 1.100$ que no le alcanzaba para cubrir sus necesidades.

Por error se había convocado a mediación solamente al hijo mayor (Walter) quien no concurrió a la primera audiencia. Este fue el primer escollo que debimos sortear ya que él se había enojado mucho con su madre por haberlo convocado solamente a él. Después supimos que era el hijo con el que mantenía la relación de mayor confrontación.

Convocamos a una próxima reunión a los tres hermanos mayores junto a Marta e Ignacio, lo hicimos por cédula de notificación y también de manera telefónica. En esta oportunidad, fue muy importante explicarles que la mediación era un espacio de diálogo, respetuoso, donde podrían sentarse cara a cara, a conversar con su madre sobre la situación familiar en la que se encontraban y que, eventualmente si llegaban a un acuerdo, éste reflejaría lo que se hubiera consensuado por todos.

A la segunda audiencia de mediación asistieron todas las personas convocadas a esos fines. Comenzamos con los tres hermanos, Walter-el hijo mayor, casado y con una bebé, quien trabajaba en una empresa-, Melisa -única hija mujer, con dos hijos, ama de casa, quien vino acompañada por su marido-y José-soltero, que vivía en la casa con su madre y trabajaba en un comercio en un régimen de extensa jornada laboral.

Los escuchamos durante un buen tiempo y pudimos advertir que no se trataba de hijos que abandonaban a su madre y a su hermano menor, sino más bien que no podían aceptar que su madre estuviera padeciendo una seria enfermedad, siendo la mujer muy joven, y que necesitara de la asistencia de ellos.

El primer trabajo estuvo referido a la aceptación de esta circunstancia, más allá de las quejas que naturalmente todo hijo puede tener sobre sus padres. Entonces tuvimos el primer acuerdo, Melisa acompañaría a su madre al médico y hablaría con la profesional sobre la enfermedad, el pronóstico, el tratamiento y los cuidados necesarios y se comprometía a transmitir esa información a sus hermanos a los fines de que todos pudieran conocer la situación que atravesaba su madre y que requería de la colaboración familiar de parte de ellos.

Por su parte, José realizaría una lista de los gastos de la casa, a saber: alquiler, servicios, alimentos, vestimenta, remedios, tarjetas etc., con el objeto de conocer los mismos y así poder afrontar los costos que devenían de las necesidades de Marta y que acordaron cubrir entre los tres hijos mayores de edad.

Asimismo, todos se comprometían a pensar, teniendo en cuenta sus horarios de trabajo y familiares, el horario en el que acompañarían a su madre du- 
rante la semana (aquí se incluyó a Ignacio) y de qué tareas se responsabilizaría cada uno de ellos a modo de colaboración en la situación familiar.

En la tercera reunión asistieron todos, con plena conciencia de la situación, y una contradictoria sensación entre la tristeza de lo inevitable (enfermedad progresiva) y la alegría de haber podido sentarse a conversar, de hecho ya habían comenzado a repartir las tareas más equitativamente.

El acuerdo contempló un aspecto económico que se pactó, teniendo en cuenta los recursos de cada uno, tareas de cuidado, de aseo en la casa, compras y comidas, aspectos relacionados a la enfermedad, etc. Pactaron también, realizar una reunión mensual para ajustar tareas y resolver cuestiones nuevas que pudieran surgir en adelante.

A Marta se la sentía muy contenta y relajada en comparación con la primera audiencia, aunque un poco demandante todavía, aspecto que trabajamos, y ella pudo escuchar a sus hijos y comprenderlos. El clima familiar había cambiado absolutamente hacia un lugar más contenedor y amoroso.

Podemos decir que la mediación no es magia, sino trabajo a conciencia que posibilita, como en estos casos, un espacio de escucha genuina y en base a los intereses de cada una de las partes que intervienen en este procedimiento.

Segundo relato: Un caso de mediación de índole penal $1^{5}$. El proceso se llevó adelante en el Centro Judicial de Mediación y se refería a una causa penal. Luego de la aceptación del cargo como mediadora y de proponer comediador, se fijó fecha y hora para la primera audiencia, a la que concurrieron ambos actores del conflicto con sus respectivos abogados.

En la primera audiencia de mediación, ya con las partes -Sra. Pérez y Sr. Gómez (los nombres son ficticios en razón de la confidencialidad)-, acompañados por sus respectivos abogados, los mediadores realizamos el discurso inicial, explicando a las partes cada uno de los principios que se establecen en la Ley $\mathrm{N}^{\circ}$ 8858; es decir la neutralidad, la confidencialidad de las audiencias (ya sean privadas o conjuntas), la comunicación directa entre las partes, la satisfactoria composición de intereses y el consentimiento informado. Asimismo, ante algunos planteos que realizaron los abogados de las partes, se explicó la implicancia de la mediación en el proceso penal. Una vez que los participantes manifestaron su deseo de trabajar en el proceso de mediación, se procedió a la firma tanto del convenio de confidencialidad como del registro de audiencias. Con ello, se solicitó a quien había realizado la denuncia -que motivó la causa penal-, que relatara la situación.

La señora Ana Pérez manifestó que se encontraba en su domicilio cuando llamaron a su puerta y ella acudió a verificar quién era. En esa oportunidad, era el padre de su hija, Juan Gómez - de quien estaba separada desde hacía ya seis

5 El relato del caso es realizado por la mediadora Karina E. Battola interviniente en una mediación realizada durante el año 2012 . 
meses-, a quien le abre la puerta, pensando que venía a retirar a Macarena -la hija de ocho años de ambos-conforme al acuerdo de familia que habían logrado en el proceso de mediación en el mes de marzo del 2012.

Juan le manifiesta que venía a buscar a la niña, pero antes necesitaba pedirle los papeles del auto (título y tarjeta verde) que Ana tenía guardados porque los necesitaba para circular, ya que él se había quedado con la posesión del automóvil desde la separación. Ella le contesta que no le entregaría los papeles porque de esa forma él podría vender el auto y se quedaría con la totalidad del dinero, sin entregarle el $50 \%$ del valor del mismo, que le correspondía por haberlo adquirido mientras estaban casados. Ante la negativa de Ana, Juan se molesta, comienza a gritarle y la situación se torna altamente conflictiva, ya que hay insultos por parte de ambos, los cuales perduran por varios minutos hasta que Juan golpea con su puño a Ana, tirándola al piso y provocándole un corte en los labios. La madre de Ana, que se encuentra en la casa, había preferido contener a Macarena en una habitación distante a la puerta de entrada para que la niña no presenciara la discusión de sus padres; escucha un golpe y llega hasta la puerta de ingreso del domicilio donde advierte que su hija se encuentra en el suelo y sangrando, razón por la cual llama a la policía. En razón de ello, realiza la denuncia y Juan es imputado por supuesto autor de lesiones leves.

Luego de esta exposición, los mediadores realizamos un parafraseo de lo narrado, quitando la carga negativa que se había expuesto en el mismo. Posteriormente, se le otorgó la palabra a Juan quien manifestó que la situación había acontecido como lo había narrado Ana, pero que en definitiva el daño no había sido tal y se encontraba muy molesto por la situación procesal en el que se encontraba y que "todo se había complicado por los papeles del auto".

A partir de entonces se desarrollaron preguntas -abiertas, circulares y reflexivas- en principio, para discernir la dimensión y la dinámica actual del conflicto y, luego, para advertir cuáles eran las expectativas que se tenían en el proceso de mediación.

En esta oportunidad, los abogados tuvieron un rol más protagónico ya que manifestaron sus posiciones al respecto y se trabajó con ellos para descubrir los intereses que sustentaban dichas posiciones. Advertidos los mismos, se estableció la necesidad de una nueva audiencia de mediación para la cual se fija fecha y hora.

En lo concerniente a la preparación dela agenda para el próximo encuentro, se efectuó un resumen de los aspectos prioritarios que surgieron en la reunión, básicamente sobre los relatos de las partes, que daban cuenta de las posiciones, y se esbozó, como hipótesis de trabajo para la segunda reunión, la cuestión concerniente a la reparación que la Sra. Pérez necesitaba de parte de su ex marido, para lo cual consideramos oportuno trabajar la re-vinculación de ellos atento a que en el futuro continuarían relacionándose por ser pareja de padres. Así, se plantearon las siguientes cuestiones: a) la necesidad de reflexionar sobre las 
distintas posibilidades que ellos tenían con respecto al automóvil del que ambos eran propietarios. b) que pensaran cómo los veía su hija en una situación de conflicto como la actual. Esta era una estrategia de trabajo a seguir que se realizaría en la próxima sesión, para trabajar la posibilidad de reparación que se advertía en los intereses de las partes.

A los fines de llevar adelante la segunda audiencia de mediación, los mediadores preparamos una agenda que contenía un resumen de los aspectos prioritarios que emergieron en la primera reunión, básicamente sobre los relatos de las partes, que reflejaban sus posiciones, y se esbozó, como hipótesis de trabajo para esta reunión, lo concerniente a la reparación que la Sra. Pérez necesitaba de parte de su ex marido. Para ello, consideramos oportuno trabajar su re-vinculación, ya que en el futuro continuarían relacionándose por ser pareja de padres.

Comenzamos entonces a trabajar en base a los intereses manifestados por las partes en el primer encuentro. Fue necesario realizar reuniones privadascon cada uno de los intervinientes. Luego se trabajó en reunión conjunta invitando a los participantes y sus abogados que asistieron con ellos, a generar una lluvia de ideas (el momento oportuno en el que se utilizó, generó quelas partes fueron creativas ylos abogados colaborativos) sobrelas posibles soluciones al conflicto en cuestión. Se acordó con los participantes la conveniencia de una próxima reunión de mediación, fijándose día y horario de la misma.

Para preparar la tercera sesión, se efectuó un resumen de los aspectos más relevantes sucedidos en la segunda audiencia y que estaban referidos a la reparación y compromiso de diálogo. Al advertirse que ambas partes estaban interesadas en la venta del auto, se planteó como tarea la posibilidad de vender el vehículo y que el dinero obtenido fuera entregado a la Sra. Pérez. Ambas partes tenían conocidos que estaban interesados en el automóvil, por lo que acordaron consultar con esas personas y transmitirles un monto de venta pactado en la presente audiencia.

Finalmente, en el tercer encuentro de mediación se trabajó en primer término con reuniones privadas con cada una de las partes. En una primera oportunidad con la Sra. Pérez y su abogada y luego con Sr. Gómez y el suyo, a los fines de que pudieran considerar el MAAN (mejor alternativa al acuerdo negociado) y el PAAN (peor alternativa al acuerdo negociado) y aplicar la técnica del abogado del diablo con cada uno de ellos. Luego se retomó la actividad en reunión conjunta con el objetivo de que se plantearan en común los puntos de acuerdo a los que las partes habían llegado. Previamente, el señor manifestó la necesidad de expresar que ya no creía que "todo se había complicado por los papeles del auto" sino que "todo se había complicado por falta de diálogo". A partir de allí, ambas partes reflexionaron sobre la necesidad de dialogar de modo pacífico sobre algunas cuestiones relativas a la disolución del vínculo conyugal -y que se encuentran pendientes al día de la fecha-para no terminar nuevamente con denuncias penales. 
Los términos del acuerdo fueron los siguientes: El señor Gómez ofreció explicaciones sobre el conflicto que derivó en la denuncia penal en su contra a la señora Pérez, quien ha escuchado las mismas. Asimismo, el señor Gómez ofrece reparar los daños ocasionados entregando la suma de dinero acordada entre ambas partes. Por su parte, la señora manifiesta dar por satisfecha su pretensión de reparación que motivó oportunamente la denuncia penal en contra del señor Gómez. En base a lo manifestado, las partes solicitan que previo cumplimiento con las formalidades de ley, el presente acuerdo sea remitido al Juzgado de Correccional para su correspondiente diligenciamiento.

A modo de reflexión, el procedimiento de mediación resultó una alternativa adecuada para abordar esta situación conflictiva, en razón del espacio propicio que se brinda para el diálogo pacífico. Asimismo, se resalta la posibilidad de asumir responsabilidades que el proceso de mediación ofrece a las partes y que resultan motivadoras de cambios para el futuro.

\section{REFLEXIONES FINALES}

La importancia de comunicar el servicio de mediación radica en que se expande el conocimiento dentro de la comunidad, espacio en el que se produce y se multiplica el conflicto. Difundir información al respecto beneficia también la comunicación interpersonal o intergrupal de los individuos en su relación social.

En muchas situaciones, la falta de información sobre la mediación -como modo pacífico de resolución de conflictos-impide quelas problemáticas que se presentan tengan rápida y efectiva solución. Asimismo, se pone de manifiesto que mal pueden losindividuos de una sociedad procurar mecanismos pacíficos de gestión de la conflictividad si no conocen la existencia de los mismos. En este sentido, se considera de vital importancia que el reemplazo de una cultura de litigio hacia una cultura de utilización de espacios de diálogo viables para abordar los conflictos, se promueva mediante la información necesaria respecto de la procedencia de estos modos no adversariales de controversias, las consecuencias de los mismos y los lugares a donde recurrir para una solicitud del servicio de mediación.

La propuesta de difusión que se realiza desde los distintos espacios, sean éstos organismos del Estado encargados de la promoción de políticas públicas, la universidad mediante actividades de extensión y divulgación científica o de práctica jurídica, y la comunidad de mediadores, implica poner a disposición de los individuos o grupos de la sociedad un conjunto de elementos necesarios para conocer sobre la pertinencia de la mediación para abordar conflictos sin necesidad de recurrir a un modo adversarial.

Sin lugar a dudas, la actividad de comunicación del servicio de mediación persigue la finalidad de sensibilizar sobre la relevancia de la utilización de este 
mecanismo y anima a recurrir al mismo para la gestión de las conflictividades en las que los individuos estén involucrados y en las que sea viable el proceso de mediación.

Finalmente, resta mencionar que el compromiso asumido en dar a conocer los modos alternativos de solución de conflictos previstos por el ordenamiento jurídico, permite que las personas estén informadas sobre distintos espacios a los que recurrir cuando necesiten dar respuesta a intereses controvertidos con otros individuos y posibilita que se descomprima el servicio adversarial, litigioso, al que se recurre para la resolución de contiendas. Así, se obtiene un mayor acceso a la justicia fortaleciendo, por una parte, el diálogo entre las personas intervinientes en una relación conflictiva y, por la otra, la responsabilidad en trabajar para la solución de la misma.

\section{REFERENCIAS}

Battola, Karina E. (2013). La cooperación en situaciones de conflicto" Córdoba: Universidad Nacional de Córdoba.

Bianchi, Roberto. (1996). Mediación prejudicial y conciliación. Comentario de la Ley 24573 y su reglamentación. Buenos Aires: Zavalia.

Calcaterra, Ruben. (2002). Mediación estratégica. Buenos Aires: Gedisa.

Eiras Nordenstahl, Ulf Christian. (2010). Mediación penal. De la práctica a la teoría. Buenos Aires: Perrot.

Entelman, Remo F. (2002). Teoría de Conflictos. Hacia un nuevo paradigma. Barcelona: Gedisa.

Higton, Elena Alvarez, Gladys S. (1995). Mediación para resolver conflictos. Buenos Aires: Ad Hoc.

Lederach, John Paul. (2000). El abecé de la paz y los conflictos. Madrid: Los libros de la Catarata.

Morin, Edgar. (1990). Introducción al pensamiento complejo. Barcelona: Gedisa

Paszucki, Samuel. (2013). Casos y cosas de mediación. Córdoba: Brujas. 